\title{
Practical Chronic Pain Management
}

Author: Patrice Forget, MD, PhD.

- Affiliation: Institute of Applied Health Sciences, Epidemiology group, School of Medicine, Medical Sciences and Nutrition, University of Aberdeen; department of Anaesthesia, NHS Grampian, Aberdeen, UK.

- Phone: +44 (0)1224 437285

- Email: forgetpatrice@yahoo.fr

- Conflicts of Interest: The author has been advisory group member for Grunenthal.

\section{Corresponding Author:}

Patrice Forget, Health Sciences Building +1, University of Aberdeen, Foresterhill Health Campus, Aberdeen, AB25 2ZD, UK.

Phone: +44 (0)1224 437285

Email: forgetpatrice@yahoo.fr

\section{Overall word count: 472}

Author's contribution: Patrice Forget: This author designed and performed the work, redacted the manuscript and approved its final version. 
Book Title: Practical Chronic Pain Management

https://www.springer.com/us/book/9783030466749

Authors: Tariq Malik, Editor

Publisher (Name, City, State): Springer Nature Switzerland AG, Gewerbestrasse 11, 6330

Cham, Switzerland

Page numbers: 388

Price (USD): \$169.99 (Hardcover); \$129.00 (eBook)

ISBN: 978-3-030-46674-9

ISBN: 978-3-030-46675-6 (eBook)

"Since a complete equation is impossible for the moment, qualitative must necessarily precede quantitative study of phenomena" (Claude Bernard, 1965). This also applies to pain medicine, as understood by Tariq Malik, editing this book. This is not a classic pain management manual, but rather an overview of how life experiences can be translated into treatment through the prism of approaches combining pragmatism and evidence-based medicine.

Tariq Malik coordinated this collaborative work, inviting experts in the field to contribute using a similar structure: starting from a clinical scenario, navigating to a diagnosis, appraising and discussing the evidence, but also managing the complexity, and sometimes the failure, of different treatments. He is Associate Professor of Anesthesia and Critical Care at the University of Chicago and has a particular interest in the management of Chronic Pain, as evidenced here. More than that, the approach he used, inviting clinicians (mostly from Chicago like him) more than scientists, indicates an interest in a patient-centered approach.

Indeed, the result is homogeneous, easy to read and follow, especially if one focuses day after day on a specific syndrome, guided by the interest of the day or the problems observed during clinical care. This gives the book the appearance of a long bedside lesson, divided into clinicallyoriented chapters, combining years of experience in the management of common (low back pain, 
fibromyalgia) and rare but critical (dysfunction of the intrathecal pump) problems in pain medicine.

The work is available as an electronic document, with a functional layout on electronic devices.

The strengths of the book relate to the practical approach, offering reasonable escalations of care plans, being realistic about the available, often limited, evidence. This could help the clinician respond with an individualized plan after evaluating the level of evidence, balancing the risk and the benefits for an individual patient and judging the appropriateness of therapy. Indeed, the experience of the patient and the clinician cannot be averaged in large series, and this book gives clues to progress in the recognition of this. If the experience-oriented format is a strength of the book, it is also a weakness. This implies that the organization does not highlight the etiology, making some chapters partially redundant, perhaps slightly discordant, or less clear than in books where the reader finds a well-identified place for specific information.

The book is primarily by clinicians for clinicians; however, this could be a very useful reference for the educator or the basic scientist as well. And, although the mention of multimodal and multidisciplinary care is consistent, the emphasis remains on medical tasks and management.

In conclusion, this book can be an excellent resource for the clinician first of all, but also for the educator and the researcher, wishing to supplement their vision and their experience of the presentations of frequent and less frequent chronic pain syndromes and possible treatments. 